\title{
Avances en enfermería informática.
}

\section{Advances in nursing informatics.}

\section{Daniel F. Condor Camara ${ }^{1}$}

A finales de los años 60 las ciencias de la salud dieron un paso importante al ingresar a la era digital, gracias a la Internet, por lo que fue favorable incorporar a la informática y al uso de las tecnologías de información y comunicación en el sector salud. En este contexto, tanto médicos como enfermeras, por ser los grupos profesionales con gran número de miembros a nivel mundial, serían los encargados de llevar esta rama de la ciencia a su crecimiento.

Así, la Informática Biomédica (IB), definida como el campo científico interdisciplinario que investiga y hace uso efectivo de los datos e información para la solución de problemas de la salud de las personas y/o comunidad; ha permitido el fortalecimiento de los sistemas de información en salud (1). En ese sentido la evolución ha sido progresiva y en la actualidad la IB cuenta con ramas, especializadas en áreas específicas como la informática clínica, la informática en salud pública, procesamiento de imágenes biomédicas, bioinformática, informática de pacientes, informática traslacional, así mismo agrupadas por grupos profesionales como la informática médica o la Enfermería Informática $(1,2)$, para este artículo nos centraremos en esta última.

Las primeras publicaciones sobre este tema fueron sobre enfermeras haciendo uso de las computadoras en su quehacer profesional, allá por el año 1966 (3). Es así como la informática en enfermería ha ido sumando innovaciones, propuestas e investigaciones que han permitido el desarrollo de esta rama. Desde entonces aparecieron más investigaciones al respecto y por ende artículos científicos, revistas específicas en la materia, por lo que se formaron grupos de trabajos y asociaciones especializadas, que empezaron a brindar programas de posgrado, hasta llegar a ser reconocida como una especialidad (4).

Desde entonces, se propusieron las primeras definiciones sobre la Enfermería Informática, pero es desde el 2015, que la "American Nurses Association" (ANA) de los Estados Unidos actualiza la definición de la Enfermería Informática (EI) como "la especialidad que integra la ciencia de enfermería con información múltiple y ciencias analíticas para identificar, definir, administrar y comunicar datos, información, conocimiento y sabiduría en la práctica de enfermería” (5).

Por su parte, en el transcurso del desarrollo de la EI se han publicado las competencias que debe tener el profesional en esta área, y según el "Technology Informatics Guiding Education Reform" (T.I.G.E.R.) de la "Healthcare Information and Management Systems Society - HIMSS (6), mencionan tres áreas primordiales: competencias básicas en computación que son los conocimientos sobre el hardware y software, el uso de la internet, la seguridad de los datos, el manejo de navegadores, entre otros conocimientos del entorno persona-computador; competencias de alfabetización informacional, que permite identificar la información adecuada y necesaria para los propósitos específicos, evaluación de la información y aplicarla correctamente; competencias de gestión de la información, que permite la recopilación, procesamiento de los datos, su presentación y comunicación para el conocimiento. 
Así, grandes eventos científicos han propiciado la difusión de los avances en EI, uno de los más reconocidos es el "International Congress on Nursing and Allied Health Informatics" organizado por "International Medical Informatics Association - Nursing Informatics (IMIA-NI)", en la edición llevado a cabo el 2018 en la ciudad de Guadalajara - México (7), se agruparon 6 temáticas principales: 1) Participación del paciente y participación ciudadana, 2) Educación, competencias y desarrollo de capacidades, 3) Soluciones, modelos y dispositivos de punto de atención, 4) Calidad, seguridad y ética, 5) Uso significativo de los sistemas de información electrónica, y 6) Análisis de Big Data y soporte de decisiones. Cada una de estas temáticas vislumbran el acontecer del desarrollo de la informática en enfermería en el contexto actual y demandante.

Si bien, el desarrollo ha ido aumentando, aun se evidencia que el conocimiento tardío de la EI impacta en la formación y el desarrollo de las enfermeras en el campo profesional (8), por lo que las nuevas enfermeras informáticas fueron capacitadas desde el posgrado (4). Así, EEUU fue el pionero en brindar esta formación, y en poco tiempo se acreditaron programas de especialización, maestrías y doctorados. Por ello y bajo el rápido desarrollo de las tecnologías que han permitido la informatización y virtualización de la atención en salud, en la última década varios países están integrando la informática en enfermería en la malla curricular en pregrado (9-11). Latinoamérica aún va detrás, especialmente en la formación de estos recursos humanos. Sin embargo, cabe precisar que algunos desarrollos regionales $(8,10)$ vislumbran los primeros pasos, pero aún hay brechas que cubrir $(12)$.

Específicamente en la región se ha visto la agrupación de diversos grupos interesados en el tema, uno de ellos y el más reconocido hasta el momento es la Red Internacional de Enfermería Informática (13), que se forma, con el objetivo de promover esta rama de la ciencia, con actividades propias, trabajando conjuntamente con perspectiva multidisciplinaria y regional. Cabe destacar, que este grupo toma como guía las recomendaciones que brindan IMIA-NI (1) y el grupo T.I.G.E.R.(6), para generar su propia agenda a fin de seguir desarrollando y promoviendo la enfermería informática.

Por su parte, la Organización Mundial de Salud promueve el uso de la informática en salud, como elemento clave para un sistema de salud eficaz (14), entonces podemos concluir que es necesario la promoción de la enfermería informática a todo nivel, visibilizar sus avances, sus competencias y el futuro de esta rama de la ciencia del cuidado, que inserte al profesional de enfermería en la era digital con competencias, metas y agendas propias para la ciencia del cuidado.

\section{REFERENCIAS BIBLIOGRÁFICAS}

1. Shortliffe EH. Biomedical Informatics in the Education of Physicians. JAMA. 2010;304(11):1227.

2. Sanches LMP, Jensen R, Monteiro MI, Lopes MHB de M. Informatics teaching in undergraduate nursing programs at Brazilian public institutions. Revista Latino-Americana de Enfermagem. 2011;19(6):138590.

3. Day PE. The International Nursing Index. The American Journal of Nursing. 1966;66(4):783.

4. Cummins MR, Gundlapalli AV, Gundlapalli AV, Murray P, Park H-A, Lehmann CU. Nursing Informatics Certification Worldwide: History, Pathway, Roles, and Motivation. Yearb Med Inform. 2016;(1):264-71.

5. Bickford CJ. Update: NI Scope and Standards of Practice, Competencies, and Certification. Stud Health Technol Inform. 2016;225:746-7.

6. Sensmeier J, Anderson C, Shaw T. International Evolution of TIGER Informatics Competencies. Stud Health Technol Inform. 2017; 232:69-76.

7. Rotegård AK, Skiba DJ, Barbosa S, Alcázar AGD, editores. Nursing informatics 2018: ICT to improve quality and safety at the point of care. Amsterdam: IOS Press; 2018.

8. Marin HF. Nursing informatics education in the South: a Brazilian experience. Yearb Med Inform. 2010; 1: 68-71.

9. Madsen I, Cummings E, Borycki EM. Current status for teaching nursing informatics in Denmark, Canada, and Australia. Stud Health Technol Inform. 2015;216:1016.

10. González ZA, Schachner MB, Tattone MA, Benítez SE. Changing educational paths in an informatics course according to the needs and expectations of nursing degree students. Stud Health Technol Inform. 2016;225:324-8.

11. O'Connor S, Gallagher J, Wamba N, Moyo C, Chirambo GB, O'Donoghue J. Establishing longterm nursing informatics capacity in Malawi, Africa. Stud Health Technol Inform. 2016; 225:1013-4.

12. Condor DF, Sanchez Alvarez K, Bidman AA. Nursing informatics training in undergraduate nursing programs in Peru. Stud Health Technol Inform. 
2018;250:81.

13. Red Internacional de Enfermería Informática. Objetivos. Red Internacional de Enfermería Informática; 2015. (Citado el 15 de setiembre del 2019) Disponible en: http://enfermeriainformaticalac. org/
14. World Health Organization. Everybody's business: strengthening health systems to improve health outcomes: WHO's frmaework for action. Geneva: World Health Organization; 2007. 